Portland State University

PDXScholar

Civil and Environmental Engineering Faculty

Publications and Presentations

$10-1-2009$

\title{
A New Look at Richardson Number Mixing Schemes for Equatorial Ocean Modeling
}

\author{
Edward D. Zaron \\ Portland State University, ezaron@pdx.edu \\ James N. Moum
}

Follow this and additional works at: https://pdxscholar.library.pdx.edu/cengin_fac

Part of the Civil and Environmental Engineering Commons

Let us know how access to this document benefits you.

\section{Citation Details}

Zaron, Edward D., James N. Moum. (2009). A New Look at Richardson Number Mixing Schemes for Equatorial Ocean Modeling. Journal of Physical Oceanography, 39, 2652-2664.

This Article is brought to you for free and open access. It has been accepted for inclusion in Civil and Environmental Engineering Faculty Publications and Presentations by an authorized administrator of PDXScholar. Please contact us if we can make this document more accessible: pdxscholar@pdx.edu. 


\title{
A New Look at Richardson Number Mixing Schemes for Equatorial Ocean Modeling
}

\author{
EDWARD D. ZARON \\ Department of Civil and Environmental Engineering, Portland State University, Portland, Oregon \\ JAMES N. MOUM \\ College of Oceanic and Atmospheric Science, Oregon State University, Corvallis, Oregon
}

(Manuscript received 28 August 2008, in final form 6 April 2009)

\begin{abstract}
A reexamination of turbulence dissipation measurements from the equatorial Pacific shows that the turbulence diffusivities are not a simple function of the gradient Richardson number. A widely used mixing scheme, the $K$-profile parameterization, overpredicts the turbulent vertical heat flux by roughly a factor of 4 in the stably stratified region between the surface mixed layer and the Equatorial Undercurrent (EUC). Additionally, the heat flux divergence is of the incorrect sign in the upper $80 \mathrm{~m}$. An alternative class of parameterizations is examined that expresses the mixing coefficients in terms of the large-scale kinetic energy, shear, and Richardson number. These representations collapse the turbulence diffusivities above and below the Equatorial Undercurrent, and a tuned version is able to reproduce the vertical turbulence heat flux within the 50-180-m depth range. Kinetic energy is not Galilean invariant, so the collapse of the data with the new parameterization suggests that oceanic turbulence responds to boundary forcing at depths well below the surface mixed layer.
\end{abstract}

\section{Introduction}

Large-scale ocean general circulation models (OGCMs) used in simulations of the earth's climate utilize a number of submodels and parameterizations to represent the transport of scalars and the dissipation of kinetic energy by turbulence. These models are necessary because the energy-containing scales of the turbulence cannot possibly be represented explicitly in global models of practicable resolution. Understanding and improving the turbulence models used in OGCMs is important because it is turbulence that determines water mass properties and the vertical buoyancy flux in the ocean interior.

The objective of this work is to reexamine microstructure measurements of turbulent kinetic energy dissipation in order to improve the parameterizations for naturally occurring shear-driven mixing. Because the ocean surface boundary layer (SBL) is relatively accessible via in situ measurement and laboratory analogs, turbulence models have been well calibrated in weakly

Corresponding author address: Edward D. Zaron, Dept. of Civil and Environmental Engineering, Portland State University, P.O. Box 751, Portland, OR 97207-0751.

E-mail: zaron@cecs.pdx.edu stratified and convectively driven boundary layers. In contrast, in the stratified ocean interior, mixing is only indirectly coupled to boundary fluxes, and turbulence is instead driven by shear, convective instability, and differential diffusion of heat and salt. Especially for sheardriven mixing, laboratory analogs do not exist that include a realistic spectrum of the background internal waves. Calibration against actual ocean data is thus necessary.

Virtually all published studies with OGCMs utilize either a two-equation turbulence model (e.g., Mellor and Yamada 1982) or the $K$-profile parameterization (KPP; Large et al. 1994). In both cases downgradient transport with an eddy or turbulence diffusivity is assumed. Let $k$ denote the turbulent kinetic energy, and $\varepsilon$ its rate of dissipation; then, the two equation models compute the momentum diffusivity (turbulent viscosity, $K_{m}$ ) with $K_{m}=C_{1} k^{2} / \varepsilon$, or some variation thereof (Saffman 1970; Burchard and Bolding 2001), and the scalar diffusivity is given by $K_{h}=\operatorname{Pr}_{t} K_{m}$, where $C_{1}$ is a dimensionless stability function and $\operatorname{Pr}_{t}$ is a turbulent Prandtl number, both possibly functions of the shear $S$ and buoyancy frequency $N$. For shear-driven mixing below the SBL, the KPP approach eliminates the explicit dependence on turbulence quantities $(k, \varepsilon)$ per se, and instead computes $K_{h}$ and $K_{m}$ from the gradient Richardson number, 
$\mathrm{Ri}=(N / S)^{2}$, alone. KPP has been implemented in all the major community OGCMs and used in hundreds of published studies (e.g., Gent et al. 1998; Li et al. 2001; Blackmon et al. 2001; Smith and Gent 2002; see Griffies et al. 2000 for a review). Because of this widespread usage, we focus on KPP with specific attention to the Ri dependence of the turbulent diffusivities below the SBL.

The use of KPP in general circulation models has generally been regarded as an improvement over the alternatives; however, this is difficult to evaluate because of a number of confounding influences. Large and Gent (1999) found that simulations of the equatorial Pacific were improved by KPP, but the model still had substantial differences compared to the observations, differences they attributed to plausible uncertainty in the air-sea fluxes. Tests of interior KPP in other sheared flow regimes (e.g., gravity-current and overflow entrainment) have not been as encouraging (e.g., Chang et al. 2005).

There has been substantial recent work with the goal of understanding and parameterizing shear-driven stratified turbulence. Baumert and Peters (2000) and Burchard and Bolding (2001) recognized key inconsistencies between existing two-equation turbulence models in this case. The role of internal waves as both a source and sink of $k$ has been considered by Dohan and Sutherland (2005) and Baumert and Peters (2004). Precisely how the energetics of the turbulence are related to existing models of the large-scale internal wave field has yet to be worked out, but there is definite support for the notion that deterministic (e.g., tidal) energy feeds a forward cascade to smaller scales that results in mixing (e.g., D'Asaro and Lien 2000; Polzin 2004). Work has also explored the performance of various turbulence models in realistic settings (Li et al. 2001; Wijesekera et al. 2003; Durski et al. 2004; Li et al. 2005; Warner et al. 2005); although, these studies have emphasized model intercomparisons, rather than direct comparisons to observations of turbulence.

Our results indicate that a reconsideration of basic $\mathrm{Ri}$-dependent mixing models is warranted. In the next section we review mixing rates and turbulence diffusivities estimated from turbulence measurements above the Equatorial Undercurrent, where shear is large and varies slowly, and we compare these with those predicted by interior KPP. The magnitude of the vertical heat flux from KPP is too high by roughly a factor of 4 , and the sign of the heat flux divergence is incorrect over the upper $80 \mathrm{~m}$ of the water column. In an attempt to fix KPP, we next apply the Buckingham pi theorem and systematically look for ways to scale the dissipation data. We find that a factor of 10 scatter in $K_{h}$ can be collapsed within a factor of 2 with the proposed model. Finally, we discuss implications of our findings and reconcile ap- parent discrepancies with respect to one of the original KPP validation studies.

\section{Does mixing depend on Ri?}

The notion that instability and turbulence is enhanced by shear and reduced by stratification is fundamental. Here, we assume that the vertical shear squared, $S^{2}=$ $(\partial u / \partial z)^{2}+(\partial v / \partial z)^{2}$, and the static stability (the square of the buoyancy frequency), $N^{2}=-(g / \rho)(\partial \rho / \partial z)-\left(g^{2} / c_{s}^{2}\right)$, are the governing quantities, where the in situ density $\rho$, sound speed $c_{s}$, and gravitational acceleration $g$ are given, and $\mathbf{V}=(u, v)$ is the horizontal velocity vector. Both laboratory observations and analytical studies suggest a relationship between the gradient Richardson number, $\mathrm{Ri}=(N / S)^{2}$, and turbulence quantities. Linear stability analysis of steady stratified shear flow (Miles 1961; Howard 1961) indicates that $\mathrm{Ri}<1 / 4$ is a sufficient condition for flow instability, while nonlinear stability analysis (Abarbanel et al. 1984) indicates that flows with larger Ri may be unstable as well. Laboratory observations of turbulence (Rohr et al. 1988) and numerical simulations (Peltier and Caulfield 2003) suggest that turbulence decays for $\mathrm{Ri}>\mathrm{Ri}_{c}$ and grows for $\mathrm{Ri}<\mathrm{Ri}_{c}$, where $\mathrm{Ri}_{c}$ is the so-called critical Richardson number. The fact that $\mathrm{Ri}_{c}$ is found to be approximately $1 / 4$ suggests that turbulence converts the minimum amount of kinetic energy to potential energy necessary to stabilize the flow, a principle that has shown some skill in predicting the turbulence dissipation rate in flows where $\mathrm{Ri}<\mathrm{Ri}_{c}$ in the ocean (Kunze et al. 1990; Peters et al. 1995). This Ri-dependent growth and decay of turbulence was used by Burchard and Bolding (2001) to calibrate a family of two-equation turbulence closure schemes.

Taylor (1931) seems to have been the first person in the English-language literature to report $K_{h}$ and $K_{m}$ versus Ri using atmospheric measurements. Later, Munk and Anderson (1948) used an Ri parameterization in a pioneering theoretical study of the ocean thermocline, and Pacanowski and Philander (1981) demonstrated that a similar parameterization produced more realistic simulations of the equatorial ocean than had been obtained previously. By confining mixing to areas of weak stratification or strong shear, the Ri-based parameterizations permitted more realistic layering, and less mixing, of the ocean circulation in coarse-resolution models.

Nonetheless, there are substantial prima facie arguments against the validity of any specific functional relationship between turbulence quantities and $\mathrm{Ri}$. The physical argument against such a relation is simply that two very different flow states (e.g., turbulent and laminar) can exist for the same value of Ri. Chang et al. (2005) emphasize that a viable turbulence parameterization must include a dependence on both Ri and the forcing 
that drives the turbulence. By definition, the three-dimensional turbulence involves energy transport through an inertial subrange, and the turbulence dissipation $\varepsilon$ is the fundamental quantity defining the rate of energy transport. Combining this reasoning with dimensional arguments led Xu et al. (2006) to suggest that turbulence cannot simply depend on $\mathrm{Ri}$, reasoning we pursue below. According to the Buckingham pi theorem (Bluman and Kumei 1989), there are not enough dimensional groups in $S^{2}, N^{2}$, and $K_{h}$ to provide a relation between $K_{h}$ and $\mathrm{Ri}$ that is invariant to the dimensional units. To make a complete, universally valid, parameterization for $K_{h}$ requires other parameters.

\section{a. Dissipation and mixing in the equatorial Pacific}

Our study is based on measurements of $\varepsilon$ obtained with microstructure shear probes on turbulence profilers during the Tropical Instability Wave Experiment (TIWE), during the fall of 1991 (Lien et al. 1995). During this experiment, two independent research groups made overlapping time series of dissipation measurements at $0^{\circ}, 140^{\circ} \mathrm{W}$, so as to validate and intercalibrate their methodologies. Simultaneous with the microstructure measurements, density was computed from temperature, salinity, and pressure (CTD) sensors on the turbulence profilers, and the large-scale horizontal velocity was measured with ship-mounted acoustic Doppler current profilers (ADCPs).

To estimate the diffusivity from $\varepsilon$, we assume a constant flux Richardson number or, equivalently, a constant mixing efficiency $\Gamma$. This (together with the assumptions of stationarity and homogeneity) is the basis for the so-called dissipation method. With these assumptions, the turbulence thermal diffusivity is estimated as (Osborn 1980)

$$
K_{h}^{\mathrm{ms}}=\Gamma \frac{\varepsilon}{N^{2}},
$$

where it has been assumed that the diffusivities of buoyancy and heat are equal. The same reasoning leads to

$$
K_{m}^{\mathrm{ms}}=(1+\Gamma) \frac{\varepsilon}{S^{2}}
$$

as an estimate of the turbulence viscosity (Peters et al. 1988). The mixing efficiency $\Gamma$ is the ratio of the vertical buoyancy flux to $\varepsilon$. We shall use the value $\Gamma=0.2$ in the calculations below (Moum 1996; Smyth et al. 2001). The superscript $\mathrm{ms}$ is a mnemonic for "microstructure."

Some care must be exercised when using Eqs. (2) and (1) because their validity requires a local balance between the production and dissipation of turbulence kinetic energy or, equivalently, that the turbulence is stationary and homogeneous, and that the turbulent pressure and velocity fields are uncorrelated. While these assumptions are not likely to be satisfied for every $\varepsilon$ measurement, it is assumed that the 20-day time series of rapidly repeated vertical profiles will have sampled an adequate number of independent turbulence events so that systematic errors will average out. Because waves may play a significant role in the momentum budget (Hebert et al. 1991b; Moum et al. 1992), our emphasis will be on the turbulence diffusivity, $K_{h}$, but $K_{m}$ comparisons are shown for completeness. Systematic errors connected with using a constant value for $\Gamma$ are considered in section 4 , below.

Computation of the diffusivity is unstable in lowgradient regions due to the finite precision of the $N^{2}$ and $S^{2}$ measurements from CTD and ADCP, respectively. Following Peters et al. (1995), the $N^{2}$ and $S^{2}$ data are spatially smoothed using a filter with a 20 -m-wavelength half-power point, and values of $K_{m}^{\mathrm{ms}}$ and $K_{h}^{\mathrm{ms}}$ are not computed within low-gradient regions $\left[N^{2}\right.$ or $S^{2}<10^{-5} \mathrm{~s}^{-2}$; Peters et al. (1988)]. Values of $N^{2}$ and $S^{2}$ used in Eqs. (2) and (1) have been temporally smoothed with a 6-h centered boxcar filter, and these averaged values are also used to compute $\mathrm{Ri}$. The results of this study are insensitive to this averaging time scale.

To make a fair comparison of microstructure-based and interior KPP diffusivities, these values are computed only for depths below the KPP-derived SBL, and we restrict our comparison to year days 310-330, a period when Wang et al. (1998) found reasonable agreement between the $\varepsilon$ values derived from a large eddy simulation (LES) and the TIWE data. This 20-day period is of added significance because it is during this time that a significant number of low-Ri events occurred below the SBL, providing the dynamic range to actually test the $\mathrm{Ri}$ parameterization. Because of changes to the thermocline connected with the passage of an equatorial Kelvin wave, the domain of applicability of interior KPP is severely restricted in space and time during the latter half of TIWE.

Figure 1 illustrates the Ri dependence of $K_{m}^{\mathrm{ms}}$ and $K_{h}^{\mathrm{ms}}$, where the data are averaged over predefined depth ranges identified by Peters et al. (1988):

1) D1: $35-65 \mathrm{~m}$ is below the SBL during the daytime, and this region generally maintains $\mathrm{Ri}<0.5$ as a consequence of coherent variations in $S^{2}$ and $N^{2}$ through the diurnal cycle (Lien et al. 1995);

2) D2: $65-100 \mathrm{~m}$ includes the so-called deep-cycle turbulence that occurs below the surface boundary layer;

3) D3: 100-125 $\mathrm{m}$ is well below the SBL and the shear is reduced approaching the EUC core near $125 \mathrm{~m}$; and

4) D4: $125-185 \mathrm{~m}$ is the deep shear layer below the EUC core.

Above the EUC core (D1-D3) there is a reduction in diffusivity with increasing Ri. Within the EUC core, $K_{m}^{\mathrm{ms}}$ 

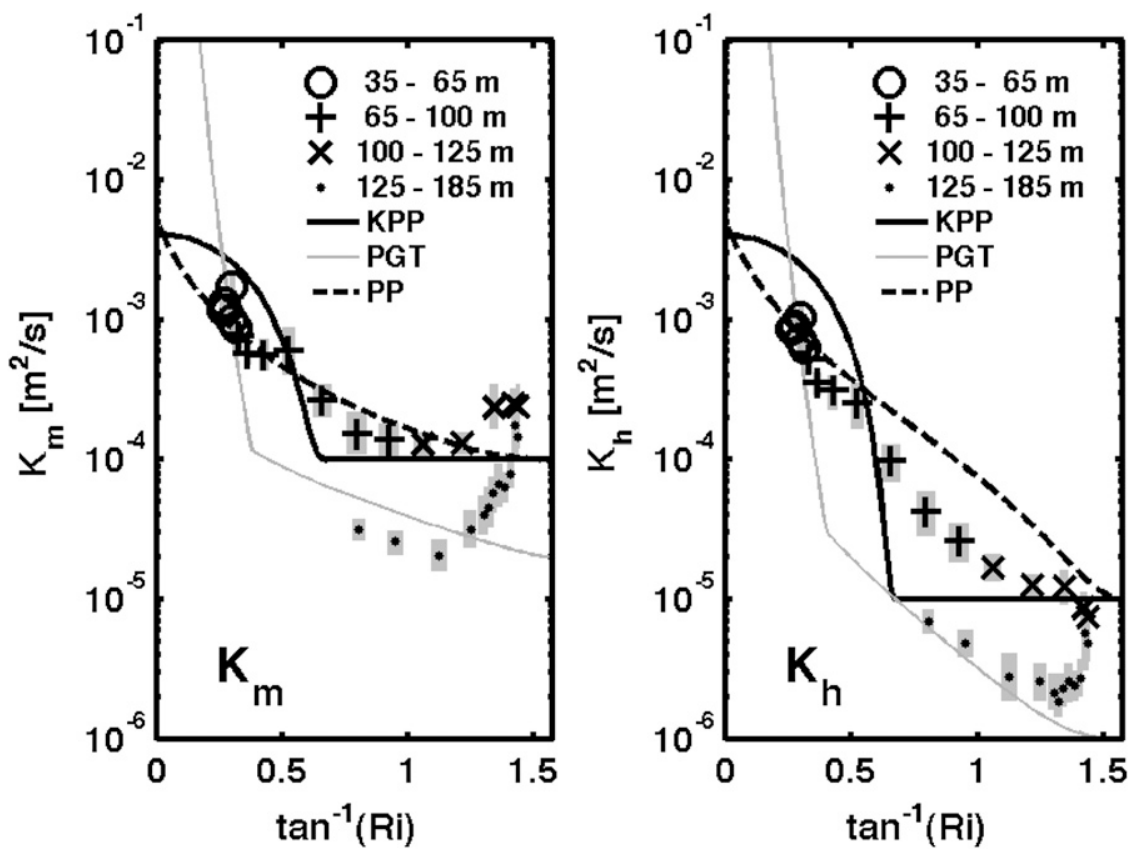

FIG. 1. Mixing coefficients: (left) eddy viscosity and (right) eddy thermal diffusivity. Dissipation method, symbols: interior KPP, dark solid line; PGT, light solid line (Peters et al. 1988); and PP, dashed line (Pacanowski and Philander 1981). Gray rectangles denote bootstrap 95\% confidence limits. Data within the surface boundary layer and low-gradient regions are excluded.

is elevated, but this may reflect a sensitivity to averaging scales of $S^{2}$, since a relation between $\varepsilon$ and the finescale shear has been found here (Peters et al. 1995). One noteworthy feature of the observations is the factor of 10 offset between diffusivities above (D3) and below (D4) the EUC core for the same range of Ri.

For reference, Fig. 2a shows the $\varepsilon$ measurements gridded at $1 \mathrm{~m} \times 1 \mathrm{~h}$ resolution. Each hourly value is the average of typically 3-10 profiles obtained from the turbulence profilers (Lien et al. 1995). Values have been blanked within the KPP-derived SBL (the gray region enclosed by the solid black line), and the nominal mixed layer depth (based on a $0.01 \Delta \rho$ criterion) is shown by a white line to emphasize that it is quite different from the SBL depth. Elevated values of $\varepsilon$ persist after, and extend below, the SBL's diurnal thinning. In Fig. $2 b$ the smoothed values of $K_{h}^{\mathrm{ms}}$ are shown; it is these values that are averaged to obtain the points in Fig. 1.

\section{b. The KPP model for Ri-dependent mixing}

KPP is based on separate models for turbulence within the ocean SBL and the ocean interior, the two models being coupled through the continuity of the turbulence diffusivities and their derivatives at the base of the boundary layer. Within the SBL, the turbulence diffusivities are parameterized by cubic functions with coefficients determined from the wind stress, buoyancy flux, and boundary conditions at the base of the boundary layer. Additionally, there is a nonlocal contribution to the scalar flux due to the effects of penetrative eddies under convective conditions. The depth of the surface boundary layer is determined by the condition that the bulk Richardson number,

$$
\operatorname{Ri}_{b}(d)=\frac{\left[B_{\text {surf }}-B(d) d\right]}{\left|\mathbf{V}_{\text {surf }}-\mathbf{V}(d)\right|^{2}+V_{t}^{2}(d)},
$$

equals a critical value, $\mathrm{Ri}_{c}=0.3$. Here, $B_{\text {surf }}$ and $\mathbf{V}_{\text {surf }}$ are near-surface values of the buoyancy and horizontal velocity, respectively; $d$ is the depth below the ocean surface, $B(d)$ is the buoyancy, $\mathbf{V}(d)$ is the velocity, and $V_{t}(d)$ is a turbulence velocity, which contributes to the boundary layer shear. To find $V_{t}$, the boundary layer shear is parameterized in terms of $N$, a generalized turbulence velocity $u^{*}$, and the distance from the ocean surface according to

$$
\left[V_{t}(d) / d\right]^{2} \propto N u^{*} / d
$$

The reader is referred to Large et al. (1994) for more details.

Below the SBL the vertical fluxes follow the downgradient transport hypothesis, with turbulence diffusivities expressed as the sum of the contributions from shear-driven 



FIG. 2. Measured and derived turbulence parameters. (a) Turbulence dissipation rate, $\varepsilon\left(\mathrm{W} \mathrm{kg}^{-1}\right)$, and turbulence diffusivity of heat $\left(\mathrm{m}^{2} \mathrm{~s}^{-1}\right)$; (b) from the dissipation method $K_{h}^{\mathrm{ms}}$; (c) from interior KPP $K_{h}^{\mathrm{kpp}}$; and (d) from a new parameterization $K_{h}^{\mathrm{rev}}$ described in section 3 . Values are blanked (gray) in the surface boundary layer computed from KPP; the mixed layer depth defined from observed density profiles is shown with the white line.

mixing, internal waves, double diffusion, and molecular diffusion. For our purposes, only the shear-driven and internal-wave components will be considered, and Eq. (3) will be used to determine the domain of applicability of the interior KPP Ri parameterization. The shear-driven part is

$$
K_{\theta}^{s} / K^{0}= \begin{cases}1, & \mathrm{Ri}<0 \\ {\left[1-\left(\mathrm{Ri}^{2} / \mathrm{Ri}_{0}\right)^{2}\right]^{3},} & 0 \leq \mathrm{Ri}<\mathrm{Ri}_{0}, \\ 0 & \text { otherwise }\end{cases}
$$

where $K^{0}=40 \times 10^{-4} \mathrm{~m}^{2} \mathrm{~s}^{-1}, \mathrm{Ri}_{0}=0.8$, and $\theta \in\{h, m\}$. Mixing caused by internal waves is assumed to combine additively with the shear-driven mixing (i.e., $K_{\theta}^{\mathrm{kpp}}=$ $K_{\theta}^{s}+K_{\theta}^{w}$, where $K_{m}^{w}=1.0 \times 10^{-4} \mathrm{~m}^{2} \mathrm{~s}^{-1}$ for momentum and $K_{h}^{w}=0.1 \times 10^{-4} \mathrm{~m}^{2} \mathrm{~s}^{-1}$ for heat). The coefficient values used here are from Large and Gent (1999).
The full KPP model of vertical mixing within and below the oceanic boundary layer has been tested using oceanic observations and LESs. In the original derivation of Large et al. (1994), the full model was calibrated against observations of upper-ocean temperature and mixed layer depth on time scales ranging from the diurnal to interannual. The qualitative and quantitative agreement with the observations is quite remarkable. Large and Gent (1999) compare the KPP model with an LES of the upper equatorial ocean (Wang et al. 1998) in order to further refine the tunable parameters and evaluate the model sensitivity. They argue that such an approach is much cleaner than can be obtained with in situ data because the vertical turbulence fluxes diagnosed from the LES can be directly compared to those from the KPP model. They find that $K^{0}$, the saturation value of the interior mixing coefficient, influences the 
temporal structure of fluxes within the boundary layer by modifying the matching conditions at the bottom of the surface boundary layer. The deep-cycle mixing is largely insensitive to $K^{0}$; it is much more sensitive to the value of $\mathrm{Ri}_{0}$. These sensitivities point to the difficulty of modeling the coupling between the surface boundary layer and the interior, an issue we shall return to in section 4.

The SBL depth must be known in order to define the domain of comparison between $K_{\theta}^{\mathrm{ms}}$ and $K_{\theta}^{\mathrm{kpp}}$. Figure 2 shows this depth as diagnosed from the surface fluxes [from surface meteorological and ocean surface data collected during TIWE; Lien et al. (1995)] using the KPP implementation in the General Ocean Turbulence Model (GOTM; Umlauf et al. 2005) by assuming a Jerlov IB water type and by restoring the GOTM temperature, salinity, and velocity fields toward the observed values with a 4-h $e$-folding time scale. The latter was necessary as GOTM is a 1-dimensional (vertical) model, and the advective fluxes and lateral pressure gradients at the equator are substantial (Wang et al. 1998).

Figure 1 shows the $K_{h}^{\mathrm{kpp}}$ and $K_{m}^{\mathrm{kpp}}$ functions together with the dissipation-based estimates of the diffusivities. Two other parameterizations are also shown for reference. The line labeled PGT shows the empirical fit obtained by Peters et al. (1988) based on a 41/2-day time series of microstructure measurements at the same location in 1984. It is interesting that their empirical function is reasonably close to the TIWE data in the D1 and deepest D4 ranges. The functional form used in the modeling study of Pacanowski and Philander (1981) is shown by the line labeled PP.

Of course, the common feature of each parameterization is a 1-1 relation between the diffusivities and $\mathrm{Ri}$, with a reduced (or constant) diffusivity for increasing $\mathrm{Ri}$. The KPP parameterization overestimates the diffusivities by a factor of $3-10$ in the $\mathrm{Ri}<0.5$ regime. For $\mathrm{Ri}>0.5$, KPP predicts a more abrupt transition to background internal wave mixing levels than is seen in the data. Below the EUC core, the observed diffusivities are almost a factor of 10 smaller than the KPP background value.

Figure $2 \mathrm{c}$ shows the temporal structure of $K_{m}^{\mathrm{kpp}}$. Values are uniformly too large above a transition depth that varies between 60 and $90 \mathrm{~m}$. The simple Ri dependence does not capture the variability in $K_{h}^{\mathrm{ms}}$ (Fig. 2b).

\section{c. Vertical fluxes}

In an OGCM it is the divergence of the turbulence fluxes that is dynamically relevant, not the values of the diffusivities per se. To assess this impact, the time-averaged vertical fluxes diagnosed from the microstructure data and interior KPP are compared in Fig. 3. The structure and magnitude of the vertical fluxes computed from both methods are quite different. Interior KPP vertical transports attain maximum values approximately 4 times larger than their dissipation method counterparts. The dissipation data predict that turbulence acts to decelerate the zonal flow uniformly at a rate of roughly $1.5 \mathrm{~cm} \mathrm{~s}^{-1}$ day $^{-1}$ between 30 and $100 \mathrm{~m}$. In contrast, the divergence of the KPP-based momentum flux would decelerate the flow at a rate of $8.3 \mathrm{~cm} \mathrm{~s}^{-1}$ day $^{-1}$ between 60 and $100 \mathrm{~m}$, and accelerate the flow above $50 \mathrm{~m}$. There is even more of a discrepancy between the inferred rates of heating. The maximum heat flux divergence is between 80 and $100 \mathrm{~m}$, where KPP obtains a value around $47 \mathrm{mK}_{\text {day }}{ }^{-1}$, roughly 5 times the rate inferred from the observations.

Note that some caution is warranted in interpreting Fig. 3 at depths shallower than about $65 \mathrm{~m}$, as the figure shows the average vertical flux below the SBL, where downgradient turbulence transport is assumed. Transport within the KPP-derived SBL is excluded from this comparison. Essentially, the fluxes shown in Fig. 3 are the average daytime fluxes, which differ substantially from the complete, 24-h, average fluxes. Even with this caveat, we believe the comparison is fair since the time scale of the shear-driven instabilities $\left(S^{-1} \approx 10 \mathrm{~min}\right)$, which interior KPP parameterizes, is short compared to the convection-free portion of the diurnal cycle $(10 \mathrm{~h})$. The total fluxes (not shown), which include the SBL contributions, are similar to those in the literature (e.g., Wang et al. 1998). We do not attempt to evaluate the KPP model for the SBL here.

Another important consideration is a difference in the method of averaging employed in Figs. 1 and 3, which has consequences for any attempt to improve the interior KPP formulation. In Fig. 1, the $K_{h}^{\mathrm{ms}}$ data are shown as averages at fixed depth, $\overline{K_{h}^{\mathrm{ms}}}(z)$, the overbar denoting the time average. For each $z$, there is an average $\overline{\operatorname{Ri}}(z)$, which is used as the argument to $K_{h}^{\mathrm{kpp}}=K_{h}^{\mathrm{kpp}} \overline{\operatorname{Ri}}(z)$ in Fig. 1. In contrast, the vertical fluxes in Fig. 3 are computed as averages of time-varying $K_{h}, \mathrm{Ri}$, and $\theta_{z}$; that is,

$$
J_{h}=-\overline{K_{h} \frac{\partial \theta}{\partial z}}=-\overline{K_{h}} \overline{\frac{\partial \theta}{\partial z}}-\overline{K_{h}^{\prime} \frac{\partial \theta^{\prime}}{\partial z}},
$$

with primes denoting fluctuations from the average. At the $20-\mathrm{m}$ vertical scale considered here, the product of the means $\left[-\overline{K_{h}}(\overline{\partial \theta} / \partial z)\right]$ dominates the mean of the product $\left[-\overline{K_{h}^{\prime}\left(\partial \theta^{\prime} / \partial z\right)}\right]$, and the concern is that $\overline{K_{h}^{\mathrm{kpp}}(\mathrm{Ri})}$ differs substantially from $K_{h}^{\mathrm{kpp}}[\overline{\mathrm{Ri}}(z)]$ shown in Fig. 1. The issue is simply that the mean of the function differs from the function of the mean:

$$
\overline{K_{h}^{\mathrm{kpp}}(\mathrm{Ri})}=K_{h}^{\mathrm{kpp}}(\overline{\mathrm{Ri}})+\frac{\sigma_{\mathrm{Ri}}^{2}}{2} \dot{K}_{h}^{\mathrm{kpp}}(\overline{\mathrm{Ri}})+\cdots,
$$


a) Zonal Stress

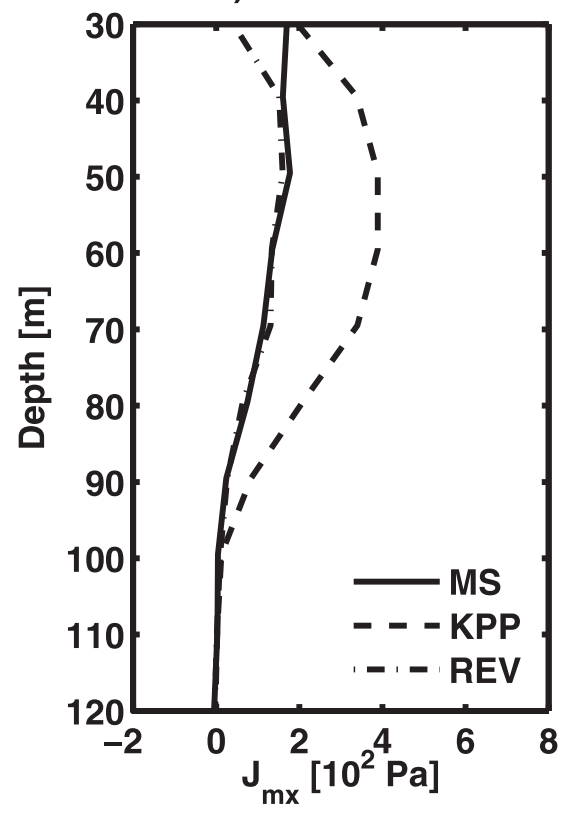

b) Heat Flux

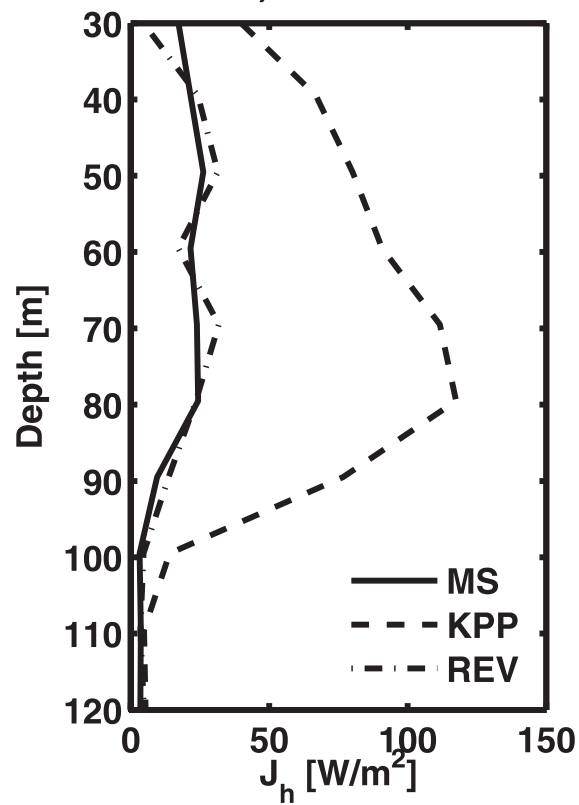

FIG. 3. Turbulence fluxes. The vertical transports of (a) zonal momentum and (b) heat (sign reversed), computed from the dissipation method (MS, solid), interior KPP (KPP, dashed), and a new parameterization [REV, dash-dot; see Eq. (9)]. Note that the vertical fluxes are those only for depths below the KPP-derived surface boundary layer, where the interior KPP Ri parameterization is nominally valid. Shallower than approximately $65 \mathrm{~m}$, the structure of the vertical flux is controlled primarily by the physics of the nighttime convective boundary layer. When the surface boundary layer flux is included (not shown), the zonal stress converges smoothly to the zonal wind stress, and the heat flux has vertical structure like that shown in Wang et al. (1998).

where $\sigma_{\mathrm{Ri}}^{2}$ is the variance of $\mathrm{Ri}$ and the overbar over $K$ denotes the differentiation with respect to Ri. In other words, variability in Ri and the curvature of the $K_{h}^{\mathrm{kpp}}(\mathrm{Ri})$ function both lead to bias in $K_{h}^{\mathrm{kpp}}(\overline{\mathrm{Ri}})$ as an estimator for $K_{h}^{\mathrm{kpp}}(\mathrm{Ri})$, and it is the latter that determines the dynamically significant vertical turbulence flux. The averaging bias explains how, in the depth range 75-125 m, the KPP diffusivity is too small $\left(K_{h}^{\mathrm{kpp}}<K_{h}^{\mathrm{ms}}\right.$, cf. Fig. 1$)$ but the KPP vertical flux is too large $\left[J_{h}^{\mathrm{kpp}}(z)>J_{h}^{\mathrm{ms}}(z)\right.$, cf. Fig. 3] even though $J_{h}$ is linear in $K_{h}$. Equation (7) shows the importance of second, and higher, moments of Ri for computing vertical fluxes when these depend nonlinearly on Ri.

\section{A new parameterization}

As a way forward for ocean modelers who wish to use a simple, but accurate, mixing scheme in the ocean interior, we consider a series of parameterizations derived from dimensional arguments, but calibrated against the TIWE observations. The basic assumption is that the turbulence is related to the stability of the flow, which we assume can be represented by the local properties of the resolved velocity and buoyancy fields. Considering the quantities that can be measured, we seek to represent the turbulent diffusivities as functions of $\left(S, N,|\mathbf{V}|^{2}, S_{z}\right)$, and the temporal modulations, $\left(|\mathbf{V}|_{t}^{2}, S_{t}, N_{t}\right)$. Dependence on the background kinetic energy, one source of energy for the shear-driven instabilities, is given by $|\mathbf{V}|^{2}$. The curvature of the velocity field is represented by $S_{z}$, which appears in theoretical expressions for the growth rate of shear-driven instabilities (Miles 1961). The time-derivative terms, $|\mathbf{V}|_{t}^{2}, S_{t}$, and $N_{t}$ contribute additional, possibly independent, time scales to the parameterization.

A straightforward application of dimensional analysis via the Buckingham pi theorem (Bluman and Kumei 1989) yields a set of five independent nondimensional parameters:

1) $\pi_{0}=N / S=\mathrm{Ri}^{1 / 2}$, the ratio of the shear to buoyancy time scales;

2) $\pi_{1}=(|\mathbf{V}| / S)\left(S / S_{z}\right)^{-1}$, the ratio of the shear length scale $(|\mathbf{V}| / S)$ to the von Kármán length $\left(S / S_{z}\right)$;

3) $\pi_{2}=\left(S / S_{t}\right) S$, the ratio of the unsteady shear $\left(S / S_{t}\right)$ to the shear time scales;

4) $\pi_{3}=\left(S / S_{t}\right)\left(|\mathbf{V}|^{2} /|\mathbf{V}|_{t}^{2}\right)^{-1}$, the ratio of the unsteady shear to unsteady kinetic energy time scales; and 

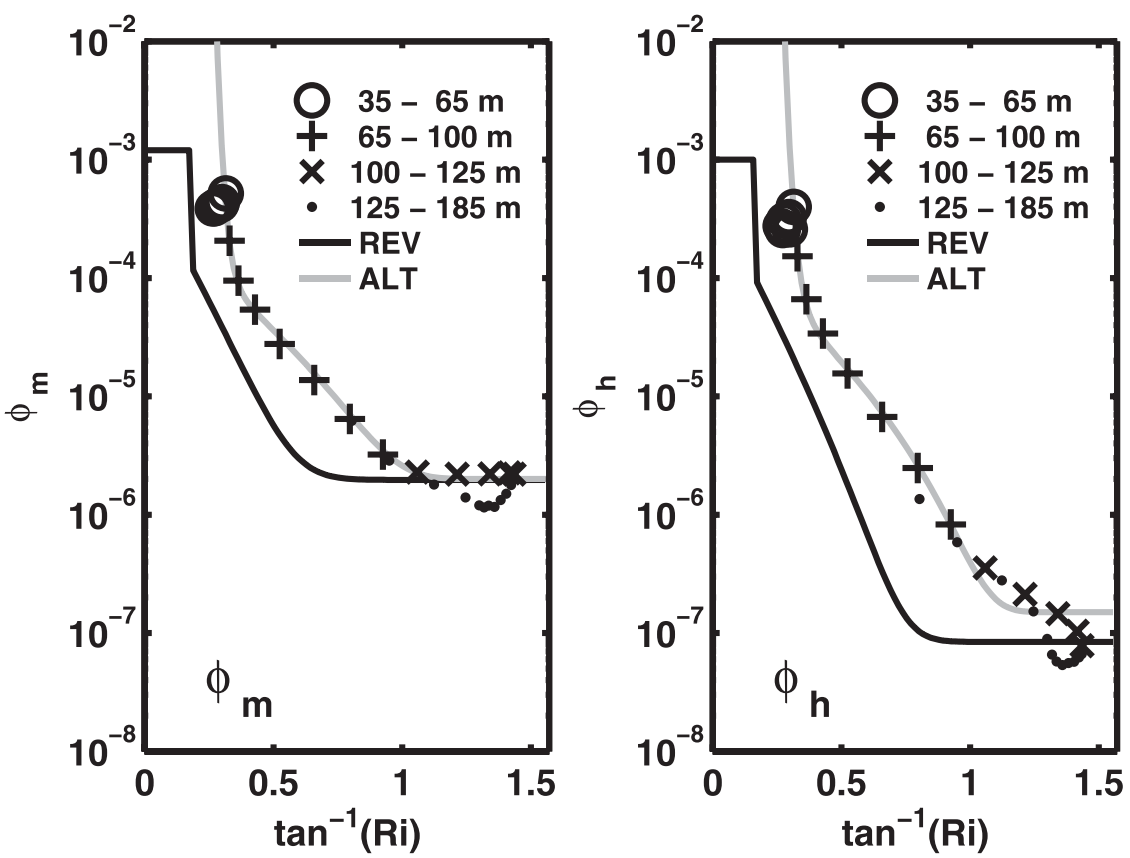

FIG. 4. Nondimensional mixing coefficients: (left) viscosity and (right) thermal diffusivity. Microstructure-derived values of $K_{\theta}^{\mathrm{ms}}$ are nondimensionalized by $\kappa_{0}=|\mathbf{V}|^{2} S^{-1}$, excluding the surface boundary layer and low-gradient regions. The solid lines indicate the proposed functional dependence described in the text: light line, $\phi_{\theta}^{\text {alt }},(8)$; and dark line, $\phi_{\theta}^{\text {rev }},(9)$.

5) $\pi_{4}=\left(N / N_{t}\right)\left(|\mathbf{V}|^{2} /|\mathbf{V}|_{t}^{2}\right)^{-1}$, the ratio of the unsteady buoyancy to unsteady kinetic energy time scales.

There are two independent groups with the units of diffusivity:

1) $\kappa_{0}=|\mathbf{V}|^{2} / S$ and

2) $\kappa_{1}=|\mathbf{V}|_{t}^{2} / S^{2}$.

The Richardson number appears in this list as $\pi_{0}^{2}$. The other $\pi_{i}$ are interpreted as ratios of macroscales that characterize flow instability or bandwidth for nonlinear interactions. Here, $\kappa_{0}$ can be regarded as a simple mixing length model with length scale $|\mathbf{V}| / S$, previously disregarded because it is not Galilean invariant (Tennekes 1989). The interpretation of $\kappa_{1}$ is unclear, and since $\kappa_{1}$ is not sign definite, it will not be considered further.

A systematic investigation of the $\pi_{i}$ parameters indicates that only $\pi_{0}$ appreciably collapses the microstructurederived data, and we are left with a simple parameterization, $K_{\theta}=\kappa_{0} \phi_{\theta}(\mathrm{Ri})$, where $\theta \in\{h, m\}$, corresponding to the scalar or momentum transport. This form retains the dependence on $\mathrm{Ri}$ but scales the intensity of the turbulence by $|\mathrm{V}|^{2} S^{-1}$. Remarkably, Fig. 4 shows that this scaling collapses the observations from above and below the EUC into a single curve (cf. with Fig. 1 from 100 to $185 \mathrm{~m}$ ).

Two functional forms are considered for $\phi_{\theta}(\mathrm{Ri})$; namely,

$$
\phi_{\theta}^{\text {alt }}=a\left(\frac{\mathrm{Ri}_{1}}{\mathrm{Ri}-\mathrm{Ri}_{1}}\right)^{\alpha}+b e^{-\beta \mathrm{Ri}}+c,
$$

and

$$
\phi_{\theta}^{\text {rev }}= \begin{cases}\phi_{\theta}^{\max } & \text { if } \mathrm{Ri} \leq \mathrm{Ri}_{2} \\ \Delta \phi_{\theta} e^{-\gamma\left(\mathrm{Ri}_{-}-\mathrm{Ri}_{2}\right)}+\phi_{\theta}^{w} & \text { otherwise. }\end{cases}
$$

The first representation $\phi_{\theta}^{\text {alt }}$ fits the observations within a factor of 2 at most depths, except in the EUC core (see gray line in Fig. 4; coefficient values in Table 1). In spite of the reasonable fit to the Ri dependence of $\phi_{\theta}=$ $K_{\theta}^{\mathrm{ms}} / \kappa_{0}$, this parameterization is useless for predicting vertical fluxes because of the bias problem in Eq. (7). The second representation $\phi_{\theta}^{\text {rev }}$ was obtained by a least squares fit to the mean $\overline{\phi_{\theta}(\mathrm{Ri})}$ computed from the microstructure data (coefficients values in Table 2). Figure 4 shows that $\phi_{\theta}^{\mathrm{rev}}$ is quite different from $\phi_{\theta}$, as is necessary to correct for the higher-order moments of Ri. By design, $\phi_{\theta}^{\text {rev }}$ yields a good fit to the vertical fluxes (Fig. 3). There is no

TABLE 1. Coefficient values for Eq. (8).

\begin{tabular}{ccccccc}
\hline \hline & $a$ & $b$ & $c$ & $\alpha$ & $\beta$ & $\mathrm{Ri}_{1}$ \\
\hline$\phi_{h}^{\text {alt }}$ & $8 \times 10^{-7}$ & $2 \times 10^{-4}$ & $1.5 \times 10^{-7}$ & 5 & -4.3 & 0.25 \\
$\phi_{m}^{\text {alt }}$ & $8 \times 10^{-7}$ & $3 \times 10^{-4}$ & $2.0 \times 10^{-6}$ & 5 & -4.0 & 0.25 \\
\hline
\end{tabular}


TABLE 2. Coefficient values for Eq. (9).

\begin{tabular}{cccccc}
\hline \hline & $\phi_{\theta}^{\max }$ & $\Delta \phi_{\theta}$ & $\phi_{\theta}^{w}$ & $\gamma$ & $\mathrm{Ri}_{2}$ \\
\hline$\phi_{h}^{\text {rev }}$ & $1.0 \times 10^{-3}$ & $9.8 \times 10^{-5}$ & $8.4 \times 10^{-8}$ & 9.86 & 0.168 \\
$\phi_{m}^{\text {rev }}$ & $1.2 \times 10^{-3}$ & $1.2 \times 10^{-4}$ & $2.0 \times 10^{-6}$ & 9.61 & 0.183 \\
\hline
\end{tabular}

particular significance to the functional form (9), except that it is piecewise continuous, saturates for small $\mathrm{Ri}$, and decays to a constant value for large Ri. It was chosen to have a minimum of free parameters while capturing the salient qualitative features of $\phi_{\theta}$. In essence, the distinction between alt (8) and rev (9) is that alt parameterizes the $\mathrm{Ri}$ dependence of the diffusivity, while rev parameterizes the Ri dependence of the vertical flux.

Figure $2 \mathrm{~d}$ compares $K_{h}^{\text {rev }}$ computed from $\phi_{h}^{\text {rev }}$. While the amplitude and spatial structure of $K_{h}^{\text {rev }}$ appear more realistic than $K_{h}^{\mathrm{kpp}}$, there is little correspondence to specific mixing events in $K_{h}^{\mathrm{ms}}$. This appears to be a limitation inherent in using a parameterization based on the large-scale gradient Richardson number, and it is consistent with the lack of correlation found by others (Peters et al. 1995).

Peters et al. (1988) suggested that the apparent structure of $K_{h}^{\mathrm{ms}}$ in their data may reflect simple depth dependence rather than a functional relationship with Ri. We have compared these alternatives using the TIWE data via a significance test with the following hypotheses:

H0: $K_{h}^{\mathrm{ms}}(z, t)$ is a function of depth alone, $\overline{K_{h}^{\mathrm{ms}}}(z)$, and

H1: $K_{h}^{\mathrm{ms}}(z, t)$ is a function of Ri and $\kappa_{0}$,

$$
\kappa_{h}^{\mathrm{ms}}=\kappa_{0} \phi_{\theta}^{\mathrm{rev}}(\mathrm{Ri}) \text {. }
$$

Since the $K_{h}^{\mathrm{ms}}$ are non-Gaussian, we use the nonparametric Spearman rank-order correlation coefficient $\left(r_{s}\right)$ to evaluate the alternatives (Conover 2001), finding $r_{s}(\mathrm{H} 0)=0.78$ and $r_{s}(\mathrm{H} 1)=0.73$. Using the bootstrap (and accounting for the reduced dof in $K_{h}^{\mathrm{ms}}$ due to the 6-h-20-m smoothing) 95\% confidence intervals are found to be $r_{s}(\mathrm{H} 0) \in[0.72,0.84]$ and $r_{s}(\mathrm{H} 1) \in[0.68,0.79]$, respectively. The overlap of these confidence intervals suggests that $\mathrm{H} 0$ and $\mathrm{H} 1$ are equally plausible.

Does the lack of a significant difference between $\mathrm{H} 0$ and H1 suggest that the diffusivity should be modeled simply as a function of depth? Probably not. Accounting for the 20-m smoothing, $\overline{K_{h}^{\mathrm{ms}}}(z)$ can be regarded as a model with seven parameters for the depth range 40-180 m. Alternately, $K_{h}^{\text {rev }}$ contains five tunable parameters; by Occam's razor it should be preferred because it is simpler. Additional evidence for the validity of the $K_{h}^{\text {rev }}$ comes from considering the correlation between the residuals $K_{h}^{\mathrm{ms}}-\overline{K_{h}^{\mathrm{ms}}}$ and $K_{h}^{\mathrm{rev}}-\overline{K_{h}^{\mathrm{ms}}}$. The Spearman rank- order correlation is $r_{s}=0.31$, which suggests that the $K_{h}^{\text {rev }}$ model explains a small, but significant, amount of the variability not explained by depth dependence alone (the critical value for $r_{s}$ is 0.14 at the $p=0.05$ significance level).

\section{Discussion}

The above comparison shows that there are large differences between the interior KPP vertical fluxes and those inferred from the dissipation method. There are questions regarding the applicability of the dissipation method for computing the turbulence diffusivities, since this already assumes a certain model for turbulence. Additionally, the disagreement between interior KPP and microstructure-derived fluxes needs some explanation, since KPP was tuned against a numerical model that nominally reproduced the main features of the diurnal cycle and compared favorably with the TIWE dissipation data (Wang et al. 1998). Here, we consider both of these issues.

\section{a. Microstructure flux estimates}

Our assessment of parameterized fluxes is based on the premise that microstructure flux estimates in (1) and (2), both of which depend linearly on $\varepsilon$, are correct. The tremendous variability in naturally occurring turbulence requires systematic and comprehensive sampling and subsequent averaging, but many such datasets now exist. From these, the following three examples illustrate the unequivocal consistency of turbulence observations with larger-scale dynamical requirements [additional examples and more detail may be found in Moum and Rippeth (2009)]:

1) Measurements of $\varepsilon$ in the upper part of convectively driven surface mixed layers are consistently equal (within $50 \%$ ) to the surface buoyancy flux (Imberger 1985; Shay and Gregg 1986; Anis and Moum 1992) and decrease linearly with depth to the mixed layer base (Anis and Moum 1994). The resultant energy balance and linear flux profile are consistent with the Monin-Obukhov scalings that represent convectively driven atmospheric mixed layers (e.g., Stull 1988).

2) Turbulence momentum flux profiles from measurements at the equator extrapolate to the surface wind stress (Dillon et al. 1989; Hebert et al. 1991a).

3) Perhaps the most significant demonstration of the veracity of microstructure flux estimates comes from comparison to dye release experiments. Vertical dye spreading represents an integrative consequence of turbulent mixing not subject to the extremes of natural 
intermittency (at least not when the distribution of the dye is sampled on annual time scales) and provides a meaningful yardstick for comparison with local microstructure flux estimates. It has been consistently found that there exists agreement of microstructure flux estimates with fluxes determined from tracer release experiments (Ledwell et al. 1993) in the main thermocline away from topographically enhanced mixing sites (Toole et al. 1994; Moum et al. 2002; Gregg 1989).

These results arise from independent efforts by multiple groups of researchers. Taken together, they offer strong evidence that microstructure estimates provide a representative means of quantifying turbulence fluxes. The mixing efficiency does vary over the lifetimes of turbulent events, and it may depend on the details of the instability causing the turbulence (Werne and Fritts 1999; Smyth and Moum 2000; Peltier and Caulfield 2003); however, there is no evidence that the mean value would differ by the factors of 4-10 necessary to account for the difference between the interior KPP and microstructurederived turbulence fluxes.

\section{b. KPP and LES}

In analyzing the diurnal cycle of turbulence in an LES of the equatorial ocean, Wang et al. (1998) found that model-derived values of $\epsilon$ agreed with the TIWE data in the 20-60-m depth range. Large and Gent (1999) tuned the KPP coefficients against these same LES simulations, so we must explain why the interior KPP scheme compares so poorly to TIWE in the present investigation.

The explanation for the discrepancy may be found by examining Fig. 16 of Wang et al. (1998), where it is shown that the eddy viscosity inferred from the LES compares poorly with the Peters et al. (1988) parameterization. In the range from $\mathrm{Ri}=0.3$ to $0.5, K_{h}$ values diagnosed from the LES are around 10 times larger than oceanic values, a result consistent with Fig. 1. In fact, Wang et al. (1998) noted the high sustained value of the flux Richardson number in their LES. In other words, the mixing efficiency of the LES is very different from that found in nature.

While it is beyond the scope of the present work to analyze the relationship between $\epsilon$ and $K_{\theta}$ in the LES, the results of Skyllingstad et al. (1999) do provide some guidance. They show that near the base of the surface boundary layer different methods of diagnosing $\varepsilon$ differ by well over a factor of 10 (see Fig. A1 in Skyllingstad et al. 1999). Turbulence quantities cannot be deduced independently of the LES subgrid-scale closure around the base of the SBL, where stratification reduces the Ozmidov scale to the grid resolution.

\section{Summary}

What was envisioned as a simple comparison of microstructure turbulence data with a widely used Richardson number mixing scheme has yielded surprising results. The comparison has revealed substantial quantitative differences, and a fresh look at the microstructure data has yielded a new parameterization for the turbulence diffusivities in terms of the gradient Richardson number, shear length scale, and the background kinetic energy. By construction, the new parameterization respects dimensional reasoning and it includes a measure of the forcing for the turbulence. Further tests are needed to examine its validity for other settings and datasets.

By design, the new parameterization $\left[\phi_{\theta}^{\text {rev }}\right.$, Eq. (9)] fits the Ri dependence of the microstructure-derived vertical flux. An alternate parameterization $\left[\phi_{\theta}^{\text {alt }}\right.$, Eq. (8)] was originally derived to explain the $\mathrm{Ri}$ dependence of the turbulence diffusivity; however, this parameterization was found to be useless for predicting vertical fluxes. Failure of the original parameterization $\left(\phi_{\theta}^{\text {alt }}\right)$ is explained by the nonlinear relationship between diffusivity and Richardson number, which causes a bias problem when we try to commute the time average with the nonlinear function of Ri.

The new parameterization is noteworthy in two regards. First, nondimensionalizing the mixing coefficient by $\kappa_{0}=|\mathbf{V}|^{2} / S$ collapses the data above and below the EUC core to within a factor of 2, whereas the original $K_{\theta}^{\mathrm{ms}}$ values differ by a factor of $5-10$. Second, it is remarkable that $\kappa_{0}$ would have this effect-this expression is not Galilean invariant. Somehow, the physics below the surface boundary layer has a preferred reference frame. We speculate that this is a consequence of the background internal wave continuum, which permits deviations from a globally homogeneous internal wave field to influence the stability of the large-scale flow. Indeed, the internal wave field above the EUC does appear to be narrowband and directional (Moum et al. 1992; Lien et al. 1995; Sun et al. 1998). The lack of Galilean invariance may also be a reflection of the fact that the largest vertical scales are, in some sense, turbulent, and the proper scaling of the dissipation follows $|\mathbf{V}|^{3} / \ell$, where $\mathbf{V}$ and $\ell$ are characteristic of the largest scales.

The discrepancies between the interior KPP scheme and the dissipation method are substantial; however, it remains to be explained how a factor of 4 error in vertical fluxes has gone unnoticed in OGCMs. There are several possibilities. First, in the time average, the interior fluxes are ultimately bounded by the surface fluxes. Perhaps the SBL component of KPP is accurate, and it has limited the consequences of errors in the interior parameterization, particularly when the diurnal 
cycle of SBL depth resets the vertical structure in the part of the water column with $\mathrm{Ri}<0.5$ during the daytime. A second possibility is that there is a negative feedback that stabilizes and systematically reduces the impact of the errors. Consider that if $J_{h}=-K_{h} \theta_{z}$ is fixed by the surface flux, a too large value of $K_{h}$ would lead to a systematically reduced $\theta_{z}$. Assuming $\rho_{z}$ and $\mathbf{V}_{z}$ are proportionally reduced, the value of Ri would increase, leading to a negative feedback (i.e., decreased $K_{h}$ ), perhaps keeping the error at an insignificant level. A third possibility is that OGCMs have chronically low vertical gradients (due to inadequate vertical resolution or excessive numerical diffusion) in the upper ocean that are compensated by the large $K_{\theta}^{\mathrm{kpp}}$ values.

It may be difficult to disentangle these alternatives in realistic OGCMs. Large and Gent (1999) found only modest improvement when KPP was used instead of a simpler Ri scheme in a three-dimensional model of the equatorial Pacific (Gent and Cane 1989), and they noted that the improved mixing scheme did not appreciably impact the model bias. More extensive model comparisons ( $\mathrm{Li}$ et al. 2001) have replicated and extended these results, but they have also shown the complexity and interrelationships among the vertical mixing parameterization and other processes. We should expect that the precise formulation and implementation of KPP will undergo revision (e.g., Danabasoglu et al. 2006) as the modeling community gains more experience. The competing impacts of surface flux errors, horizontal mixing, SBL modeling, and interior mixing models certainly deserve more attention.

The fundamental physical problem with relating diffusivity to $\mathrm{Ri}$ alone is that $\mathrm{Ri}$ cannot predict the intensity of the mixing, it can only constrain the stability of the flow. As mentioned in the introduction, a valid mixing parameterization should account for the intensity of the forcing as well as the stability of the flow (Chang et al. 2005). A broader criticism of $\mathrm{Ri}$-based approaches is that the flow instabilities we are attempting to parameterize have their genesis at scales smaller than can be resolved by coarse-resolution ocean models. At the resolution of the numerical models, Ri may not even characterize the stability of the flow. This is a central argument of Peters et al. (1995), who find that the 20-m $\mathrm{Ri}$ is poorly correlated with mixing parameters, especially compared with parameterizations based on the finescale shear. Likewise, Moum et al. (2003) suggested that turbulence in propagating solitary waves is initiated by shear at $10-\mathrm{cm}$ scales, a scale approximately two orders of magnitude smaller than the 20 -m vertical scale of the solitons. The comments of Chang et al. (2005) should be broadened so that the "strength of the forcing" refers not only to the processes that maintain the mean shear, but also the processes that maintain the internal waves that trigger the turbulence.

There is abundant observational data to challenge any parameterization of upper-ocean mixing. For example, Johnson and Luther (1994) conducted an analysis of the momentum budget between $4^{\circ} \mathrm{S}$ to $10^{\circ} \mathrm{N}$ and $150^{\circ}$ to $158^{\circ} \mathrm{W}$ using data collected during the North Pacific Shuttle Experiment's (NORPAX) Hawaii to Tahiti Shuttle Experiment. They found that the meridional structure of the inferred turbulence viscosity is inconsistent with interior KPP and other Ri schemes: as one moves off the equator, the values of $K_{m}$ and Ri both increase at all depths above $80 \mathrm{~m}$. Other complexity is apparent in LES and direct numerical simulations. Wang and Muller (2002) conclude their study with the proviso that "equatorial turbulence cannot be adequately parameterized based on the value of Ri alone." Although their simulations essentially cover just one 8-h realization of convective mixing in the presence of shear, they find that turbulence generated in the marginally stable region below the surface mixed layer results from both mean shear and transient internal waves. These results are consistent with observations that show that waves (not turbulence) carry a significant fraction of the downward momentum flux in the upper ocean (Hebert et al. 1991b). Perhaps there will be a compromise between completely empirical parameterizations, such as those developed here, and higher-order closure schemes that will be capable of parameterizing the role of waves in shear-driven turbulence (e.g., Baumert and Peters 2004).

Acknowledgments. The authors benefited from the helpful criticism of Hartmut Peters and William Large, who reviewed an early version of this manuscript. Conversations with Bill Smyth, Jonathan Nash, and Andrew Bennett also contributed to this research. EDZ was supported by Grant OCE0623540 from the National Science Foundation. JNM was supported by Grant 0424133 from the National Science Foundation.

\section{REFERENCES}

Abarbanel, H. D., D. D. Holm, J. E. Marsden, and T. Ratiu, 1984: Richardson number criterion for the nonlinear stability of $3 \mathrm{D}$ stratified flow. Phys. Rev. Lett., 52, 2352-2355.

Anis, A., and J. N. Moum, 1992: The superadiabatic surface layer of the ocean during convection. J. Phys. Oceanogr., 22, 12211227.

— rate in the upper ocean during convection. J. Phys. Oceanogr., 24, 2142-2155.

Baumert, H., and H. Peters, 2000: Second-moment closures and length scales for weakly stratified turbulent shear flows. J. Geophys. Res., 105 (C3), 6453-6468. 
— and - 2004: Turbulence closure, steady state, and collapse into waves. J. Phys. Oceanogr., 34, 505-512.

Blackmon, M., and Coauthors, 2001: The Community Climate System Model. Bull. Amer. Meteor. Soc., 82, 2357-2376.

Bluman, G., and S. Kumei, 1989: Symmetries and Differential Equations. Applied Mathematical Sciences, Vol. 81, SpringerVerlag, 412 pp.

Burchard, H., and K. Bolding, 2001: Comparative analysis of four second-moment turbulence closure models for the oceanic mixed layer. J. Phys. Oceanogr., 31, 1943-1968.

Chang, Y. S., X. Xu, T. M. Özgökmen, E. P. Chassignet, H. Peters, and P. F. Fischer, 2005: Comparison of gravity current parameterizations and calibration using a high-resolution 3D nonhydrostatic spectral element model. Ocean Modell., 10, 342-368.

Conover, W. J., 2001: Practical Nonparametric Statistics. 3rd ed. John Wiley, $584 \mathrm{pp}$.

Danabasoglu, G., W. G. Large, J. J. Tribbia, P. R. Gent, B. P. Briegleb, and J. C. Mc Williams, 2006: Diurnal coupling in the tropical oceans of CCSM3. J. Climate, 19, 2347-2365.

D'Asaro, E. A., and R. Lien, 2000: The wave-turbulence transition for stratified flows. J. Phys. Oceanogr., 30, 1669-1678.

Dillon, T. M., J. N. Moum, T. K. Chereskin, and D. R. Caldwell, 1989: Zonal momentum balance at the equator. J. Phys. Oceanogr., 19, 561-570.

Dohan, K., and B. R. Sutherland, 2005: Numerical and laboratory generation of internal waves from turbulence. Dyn. Atmos. Oceans, 40, 43-56.

Durski, S. M., S. M. Glenn, and D. B. Haidvogel, 2004: Vertical mixing schemes in the coastal ocean: Comparison of the level 2.5 Mellor-Yamada scheme with an enhanced version of the K profile parameterization. J. Geophys. Res., 109, C01015, doi:10.1029/2002JC001702.

Gent, P. R., and M. A. Cane, 1989: A reduced-gravity, primitive equation model of the upper equatorial ocean. J. Comput. Phys., 81, 444-480.

_ , F. O. Bryan, G. Danabasoglu, S. C. Doney, W. R. Holland, W. G. Large, and J. C. McWilliams, 1998: The NCAR Climate System Model global ocean component. J. Climate, 11, 12871306.

Gregg, M. C., 1989: Scaling turbulent dissipation in the thermocline. J. Geophys. Res., 94 (C7), 9686-9698.

Griffies, S. M., and Coauthors, 2000: Developments in ocean climate modelling. Ocean Modell., 2, 123-192.

Hebert, D., J. N. Moum, and D. R. Caldwell, 1991a: Does ocean turbulence peak at the equator?: Revisited. J. Phys. Oceanogr., 21, 1690-1698.

,-- C. A. Paulson, D. R. Caldwell, T. K. Chereskin, and M. J. McPhaden, 1991b: The role of the turbulent stress divergence in the equatorial Pacific momentum balance. J. Geophys. Res., 96, 7127-7136.

Howard, L. N., 1961: A note on the paper of John W. Miles. J. Fluid Mech., 10, 509-512.

Imberger, J., 1985: The diurnal mixed layer. Limnol. Oceanogr., 30, 737-770.

Johnson, E. S., and D. S. Luther, 1994: Mean zonal momentum balance in the upper and central equatorial Pacific Ocean J. Geophys. Res., 99 (C4), 7689-7705.

Kunze, E., A. Williams III, and M. G. Briscoe, 1990: Observations of shear and stability from a neutrally buoyant float. $J$. Geophys. Res., 95, 18 127-18 142.

Large, W. G., and P. R. Gent, 1999: Validation of vertical mixing in an equatorial ocean model using large eddy simulations and observations. J. Phys. Oceanogr., 29, 449-464.
_ J. C. McWilliams, and S. C. Doney, 1994: Oceanic vertical mixing: A review and a model with a nonlocal boundary layer parameterization. Rev. Geophys., 32, 363-403.

Ledwell, J., A. Watson, and C. Law, 1993: Evidence for slow mixing across the pycnocline from an open-ocean tracer-release experiment. Nature, 364, 701-703.

Li, M., L. Zhong, and W. C. Boicourt, 2005: Simulations of Chesapeake Bay estuary: Sensitivity to turbulence mixing parameterizations and comparison with observations. J. Geophys. Res., 110, C12004, doi:10.1029/2004JC002585.

Li, X., Y. Chao, J. C. McWilliams, and L. Fu, 2001: A comparison of two vertical-mixing schemes in a Pacific Ocean general circulation model. J. Climate, 14, 1377-1398.

Lien, R. C., D. R. Caldwell, M. C. Gregg, and J. N. Moum, 1995: Turbulence variability at the equator in the central Pacific at the beginning of the 1991-1993 El Niño. J. Geophys. Res., 100 (C4), 6881-6898.

Mellor, G. L., and T. Yamada, 1982: Development of a turbulence closure model for geophysical fluid problems. Rev. Geophys., 20, 851-875.

Miles, J. W., 1961: On the stability of heterogeneous shear flows. J. Fluid Mech., 10, 496-508.

Moum, J. N., 1996: Efficiency of mixing in the main thermocline. J. Geophys. Res., 101 (C5), 12 057-12 069.

, and T. P. Rippeth, 2009: Do observations adequately resolve the natural variability of oceanic turbulence? J. Mar. Syst., 77, 409-417.

-, D. Herbert, C. Paulson, and D. Caldwell, 1992: Turbulence and internal waves at the equator. Part I: Statistics from towed thermistors and a microstructure profiler. J. Phys. Oceanogr., 22, 1330-1345.

, D. Caldwell, J. D. Nash, and G. Gunderson, 2002: Observations of boundary mixing over the continental slope. J. Phys. Oceanogr., 32, 2113-2130.

—, D. M. Farmer, W. D. Smyth, L. Armi, and S. Vagle, 2003: Structure and generation of turbulence at interfaces strained by internal solitary waves propagating shoreward over the continental shelf. J. Phys. Oceanogr., 33, 2093-2112.

Munk, W. H., and E. R. Anderson, 1948: Notes on a theory of the thermocline. J. Mar. Res., 7, 276-295.

Osborn, T., 1980: Estimates of the local rate of vertical diffusion from temperature measurements. J. Phys. Oceanogr., 10, 83-89.

Pacanowski, R., and S. Philander, 1981: Parameterization of vertical mixing in numerical models of tropical oceans. J. Phys. Oceanogr., 11, 1443-1451.

Peltier, W. R., and C. P. Caulfield, 2003: Mixing efficiency in stratified shear flows. Annu. Rev. Fluid Mech., 35, 135-167.

Peters, H., M. C. Gregg, and J. M. Toole, 1988: On the parameterization of equatorial turbulence. J. Geophys. Res., 93 (C2), $1199-1218$

- - , and T. B. Sanford, 1995: On the parameterization of equatorial turbulence: Effect of finescale variations below the range of the diurnal cycle. J. Geophys. Res., 100 (C9), 18333 18348 .

Polzin, K., 2004: A heuristic description of internal wave dynamics. J. Phys. Oceanogr., 34, 214-230.

Rohr, J. J., E. C. Itsweire, K. N. Hellland, and C. W. Van Atta, 1988: Growth and decay of turbulence in a stably stratified shear flow. J. Fluid Mech., 195, 77-111.

Saffman, P. G., 1970: A model for inhomogeneous turbulent flow. Proc. Roy. Soc. London, 317A, 417-433.

Shay, T., and M. Gregg, 1986: Convectively driven turbulent mixing in the upper ocean. J. Phys. Oceanogr., 16, 1777-1798. 
Skyllingstad, E. D., W. D. Smyth, J. N. Moum, and H. Wijesekera, 1999: Upper-ocean turbulence during a westerly wind burst: A comparison of large-eddy simulations and microstructure measurements. J. Phys. Oceanogr., 29, 5-28.

Smith, R., and P. Gent, 2002: Reference Manual for the Parallel Ocean Program (POP). Tech. Doc. LAUR-02-2484, Los Alamos National Laboratory, Los Alamos, NM 74 pp.

Smyth, W. D., and J. N. Moum, 2000: Length scales of turbulence in stably stratified mixing layers. Phys. Fluids, 12, 1327-1342.

- — - and D. R. Caldwell, 2001: The efficiency of mixing in turbulent patches: Inferences from direct simulations and microstructure observations. J. Phys. Oceanogr., 31, 1969-1992.

Stull, R. B., 1988: An Introduction to Boundary Layer Meteorology. Kluwer, 666 pp.

Sun, C., W. D. Smyth, and J. N. Moum, 1998: Dynamic instability of stratified shear flow in the upper equatorial Pacific. J. Geophys. Res., 103, 10 323-10 338.

Taylor, G. I., 1931: Internal waves and turbulence in a fluid of variable density. The Scientific Papers of Sir Geoffrey Ingram Taylor. Vol. II, Meteorology, Oceanography, and Turbulent Flow, G. K. Batchelor, Ed., Cambridge University Press, 240246.

Tennekes, H., 1989: Two- and three-dimensional turbulence. Lecture Notes on Turbulence, J. R. Herring and J. C. McWilliams, Eds., World Scientific, 1-73.
Toole, J. M., K. L. Polzin, and R. W. Schmitt, 1994: Estimates of diapycnal mixing in the abyssal ocean. Science, 264, 1120-1123.

Umlauf, L., H. Burchard, and K. Bolding, 2005: GOTM sourcecode and test case documentation. Version 3.2. Meereswiss. Ber. Tech. Rep. 63, Baltic Sea Research Institute, Warnemünde, Germany, 360 pp.

Wang, D., and P. Muller, 2002: Effects of equatorial undercurrent shear on upper-ocean mixing and internal waves. J. Phys. Oceanogr., 32, 1041-1057.

— J. C. McWilliams, and W. G. Large, 1998: Large-eddy simulation of the diurnal cycle of deep equatorial turbulence. J. Phys. Oceanogr., 28, 129-148.

Warner, J. C., C. R. Sherwood, H. G. Arango, and R. P. Signell, 2005: Performance of four turbulence closure models implemented using a generic length scale method. Ocean Modell., 8, 81-113.

Werne, J., and D. C. Fritts, 1999: Stratified shear turbulence: Evolution and statistics. Geophys. Res. Lett., 26, 439-442.

Wijesekera, H. W., J. S. Allen, and P. A. Newberger, 2003: Modeling study of turbulent mixing over the continental shelf: Comparison of turbulent closure schemes. J. Geophys. Res., 108, C3103, doi:10.1029/2001JC001234.

Xu, X., Y. S. Chang, H. Peters, T. M. Özgökmen, and E. P. Chassignet, 2006: Parameterization of gravity current entrainment for ocean circulation models using a high-order 3D nonhydro-static spectral element model. Ocean Modell., 14, $19-44$. 
Copyright of Journal of Physical Oceanography is the property of American Meteorological Society and its content may not be copied or emailed to multiple sites or posted to a listserv without the copyright holder's express written permission. However, users may print, download, or email articles for individual use. 